\title{
Aortic Root Abscess Presenting as Syncope and Substernal Chest Pain: Case Report and Literature Review
}

ISSN: 2639-0531

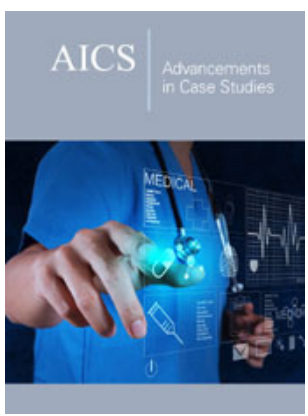

*Corresponding author: Marjan Alidoost, Department of Medicine, USA

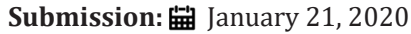

Published: 㘹 January 24, 2020

Volume 2 - Issue 3

How to cite this article: Conte GA, Marjan A, John M, Hossain M. Aortic Root Abscess Presenting as Syncope and Substernal Chest Pain: Case Report and Literature Review. Adv Case Stud.2(3). AICS.000537.2020.

DOI: 10.31031/AICS.2020.02.000537

Copyright@ Marjan Alidoost, This article is distributed under the terms of the Creative Commons Attribution 4.0 International License, which permits unrestricted use and redistribution provided that the original author and source are credited.
Conte GA, Marjan Alidoost*, John Mikhail and Hossain MA

Department of Medicine, USA

\begin{abstract}
Background: Aortic root abscess (ARA) is a serious complication of infective endocarditis (IE) which requires urgent/emergent surgery. ARA has high intra-operative mortality, estimated to be as high as $12 \%$. ARA does not always have specific symptoms, so a high index of suspicion from clinicians is key for diagnosis.

Case presentation: A 53-year-old male with a past medical history of type A aortic dissection (AD) status post repair one year prior to presentation, chronic foot wound and hypertension presented to the Emergency Department (ED) with substernal chest pain radiating to his back and neck. He also reported an episode of syncope at home. In ED, he was found to be hypoxic. Initial Computed Tomography chest was done without contrast due to elevated creatinine and was negative for pulmonary embolism (PE). Ventilation/perfusion scan showed low probability of PE and blood cultures came back positive for grampositive cocci, eventually specified as methicillin resistant staphylococcus aureus (MRSA). Repeat CT chest with contrast showed fluid collection near the aortic arch and he was diagnosed with an ARA. He was initially treated with drainage of the abscess by interventional radiology (IR) and was transferred to another facility for surgical management.
\end{abstract}

Conclusion: Although infective endocarditis (IE) is routinely managed with medical therapy alone, ARA is an indication for surgery. Patients with ARA are often critically ill and at risk for many disastrous complications, including acute congestive heart failure (CHF), septic shock, fistula formation, and involvement of the mitral valve. It is integral to promptly diagnose this condition so patients may receive proper treatment and care.

Keywords: Infective endocarditis (IE); Aortic root abscess (ARA); Peri-Annular abscess

\section{Introduction}

Infective endocarditis (IE) is a disease with a high morbidity and mortality rate, with 1-year mortality estimated at $40 \%$ [1]. Some of the more common complications include congestive heart failure (CHF) and arrhythmia [2]. Another feared complication of IE is abscess development, especially abscess which involves the aortic root. Aortic root abscess formation is an indication for surgical treatment. Aortic root abscess (ARA), sometimes called peri-annular abscess, in particular, has a high operative mortality, estimated to be $12 \%$, which has largely been attributed to the fact that the patients who develop ARA are critically ill [3]. ARA can manifest in a myriad of ways, ranging from the classic development of a new murmur to new-onset atrioventricular (AV) block and to less common presentations such as ischemia from coronary artery compression [4-6]. Since ARA can prove a challenging diagnosis, and these patients are often critically ill requiring emergent surgery and hemodynamic support, prompt diagnosis is imperative [4]. In this case report, we present a patient who presented with chest pain, shortness of breath and hypoxia and was initially thought to have a pulmonary embolism (PE), but was subsequently found to have an ARA.

\section{Case Presentation}

A 53-year-old Caucasian male with a history of type A aortic dissection status post repair with 24 millimeters $(\mathrm{mm})$ and $28 \mathrm{~mm}$ Gel-weave grafts two years ago and psoriasis on dupilumab presented to the Emergency Department with complaints of fevers, chills, substernal chest discomfort radiating to the back as well as two syncopal episodes at home. The patient denied recent weight loss, nausea, vomiting or abdominal pain. Upon initial physical 
examination, the patient was having conversational dyspnea and in respiratory distress requiring accessory muscle use. A new grade II holosystolic murmur with a radiation to the axilla was appreciated on the cardiac exam. Lungs were clear to auscultation bilaterally. Psoriatic plaques were present on the dorsal aspects of both feet. The remainder of the examination was within normal limits. His vital signs were as follows: blood pressure: 88/47 millimeters of mercury (mmHg); heart rate: 110 beats per minute; respiratory rate: 22/minute; body temperature: 99.3 degrees Fahrenheit; oxygen saturation: $91 \%$ room air.

The results of a complete metabolic panel Table 1 showed elevation of serum blood urea nitrogen and creatinine at $26 \mathrm{mg} /$ $\mathrm{dL}$ and $2.61 \mathrm{mg} / \mathrm{dL}$ (baseline creatinine $0.93 \mathrm{mg} / \mathrm{dL}$ ) respectively along with decreased bicarbonate at $23 \mathrm{mmol} / \mathrm{L}$. The patient was furthermore found to have an elevated lactic acid value of $2.2 \mathrm{mmol} / \mathrm{L}$. In addition, complete blood count Table 1 showed a white blood cell count of $10.7 \mathrm{~K} / \mathrm{uL}$ with bandemia of $37 \%$. Due to acute kidney injury, the contrast was not administered for the initial computed tomography (CT) scan of the chest, abdomen and pelvis, which showed evidence of prior ascending thoracic aortic repair with a central displacement of atherosclerotic calcifications within the distal abdominal aorta (Figure 1). The patient was subsequently admitted to the intensive care unit for hemodynamic instability in the setting of sepsis, for which he received adequate fluid resuscitation with intravenous (IV) normal saline followed by maintenance fluids as well as an empirical broad spectrum antibiotics Within the first

Table 1: Laboratory results of the patient.
24 hours, 2 separate blood cultures yielded positive for methicillin resistant staphylococcus aureus (MRSA) and the patient was resumed on IV Vancomycin. Ventilation and perfusion scan were negative and transthoracic echocardiogram showed mildly reduced ejection fraction of 51-54\%, mildly increased left ventricular wall thickness and hypokinesis of the apex, apical anterior and apical lateral segments. The aortic root was not well visualized. On hospital day two, a transesophageal echocardiogram (TEE) was performed at the bedside and resulted in negative for vegetation. This was complicated by the patient being intubated and sedated post-procedure due to acute hypoxic respiratory failure. As the patient's renal function improved to baseline (Table 1), he underwent CT angiography of the chest to rule out of an aortic graft leak, which showed increased fluid surrounding the ascending thoracic aorta and anterior to the trachea without extravasation of the dye (Figure 2). The true and false lumens of prior dissection in the arch of the aorta were also visualized. Cardiothoracic surgery was thus immediately consulted and conversed the greater than $50 \%$ operative risk of surgical management. The patient subsequently underwent redo-sternotomy and drainage of the peri-aortic abscess cavity. Intra-operative cultures of the abscess collection and ascending aorta peri-graft tissue were positive for MRSA. The patient was then transferred to a specialized facility for homograft aortic root replacement with extra-anatomic bypass and revascularization. Communication with the patient's father by telephone several months after transfer from our facility revealed that the patient was alive and in stable condition.

\begin{tabular}{|c|c|c|c|}
\hline & $\begin{array}{c}\text { Result } \\
\text { Hospital Day } 1\end{array}$ & $\begin{array}{c}\text { Results } \\
\text { Hospital Day } 2\end{array}$ & Reference Range \\
\hline \multicolumn{4}{|c|}{ Complete metabolic panel } \\
\hline Sodium & $135 \mathrm{mmol} / \mathrm{L}$ & $136 \mathrm{mmol} / \mathrm{L}$ & $136-145 \mathrm{mmol} / \mathrm{L}$ \\
\hline Potassium & $4.0 \mathrm{mmol} / \mathrm{L}$ & $3.3 \mathrm{mmol} / \mathrm{L}$ & $3.5-5.2 \mathrm{mmol} / \mathrm{L}$ \\
\hline Chloride & $102 \mathrm{mmol} / \mathrm{L}$ & $109 \mathrm{mmol} / \mathrm{L}$ & $96-110 \mathrm{mmol} / \mathrm{L}$ \\
\hline Bicarbonate & $23 \mathrm{mmol} / \mathrm{L}$ & $20 \mathrm{mmol} / \mathrm{L}$ & $24-31 \mathrm{mmol} / \mathrm{L}$ \\
\hline Calcium & $8.8 \mathrm{mg} / \mathrm{dL}$ & $8.6 \mathrm{mg} / \mathrm{dL}$ & $8.5-10.5 \mathrm{mg} / \mathrm{dL}$ \\
\hline Magnesium & $1.5 \mathrm{mg} / \mathrm{dL}$ & $1.6 \mathrm{mg} / \mathrm{dL}$ & $1.3-2.5 \mathrm{mg} / \mathrm{dL}$ \\
\hline Phosphorous & $3.2 \mathrm{mg} / \mathrm{dL}$ & $2.2 \mathrm{mg} / \mathrm{dL}$ & $2.5-4.6 \mathrm{mg} / \mathrm{dL}$ \\
\hline Bun & $26 \mathrm{mg} / \mathrm{dL}$ & $8 \mathrm{mg} / \mathrm{dL}$ & $5.0-25 \mathrm{mg} / \mathrm{dL}$ \\
\hline Creatinine & $2.61 \mathrm{mg} / \mathrm{dL}$ & $0.84 \mathrm{mg} / \mathrm{dL}$ & $0.61-1.24 \mathrm{mg} / \mathrm{dL}$ \\
\hline eGFR & $26 \mathrm{~mL} / \mathrm{min}$ & $>60 \mathrm{~mL} / \mathrm{min}$ & $>60 \mathrm{~mL} / \mathrm{min}$ \\
\hline Lactic acid & $2.2 \mathrm{mmol} / \mathrm{L}$ & & $0.5-2.0 \mathrm{mmol} / \mathrm{L}$ \\
\hline \multicolumn{4}{|c|}{ Complete blood count } \\
\hline WBC & $10.7 \mathrm{~K} / \mathrm{uL}$ & $9.6 \mathrm{~K} / \mathrm{uL}$ & $4.5-11.0 \mathrm{~K} / \mathrm{uL}$ \\
\hline Hemoglobin & $14.3 \mathrm{gm} / \mathrm{dL}$ & $12.2 \mathrm{gm} / \mathrm{dL}$ & $12.0-17.5 \mathrm{gm} / \mathrm{dL}$ \\
\hline Hematocrit & $41.3 \%$ & $35.3 \%$ & $36.0-53.0 \%$ \\
\hline MCV & 87.3fL & $85.9 \mathrm{fL}$ & $80.0-100 \mathrm{fL}$ \\
\hline $\mathrm{MCH}$ & $30.2 \mathrm{PG}$ & $29.7 \mathrm{PG}$ & $25.0-35.0 \mathrm{PG}$ \\
\hline Platelet Count & $145 \mathrm{~K} / \mathrm{uL}$ & $111 \mathrm{~K} / \mathrm{uL}$ & $140-450 \mathrm{~K} / \mathrm{uL}$ \\
\hline \multicolumn{4}{|c|}{ Differential type } \\
\hline Band Neutrophil & $37 \%$ & $28 \%$ & $5-11 \%$ \\
\hline
\end{tabular}




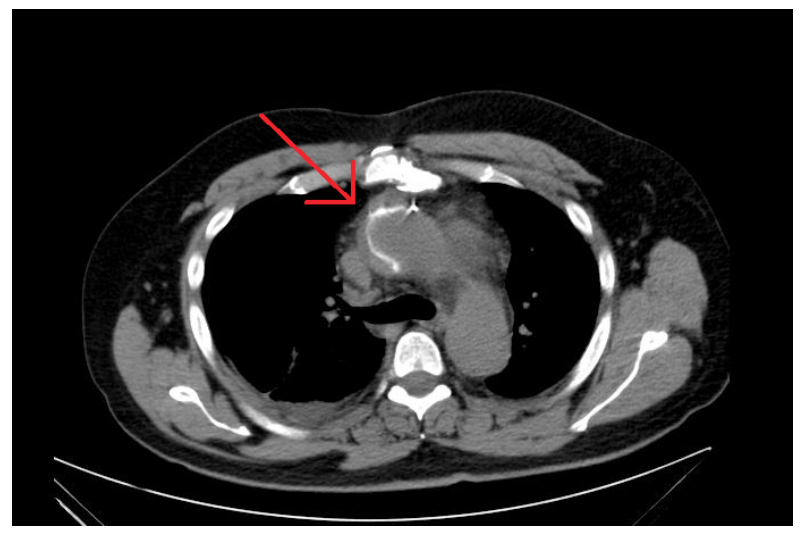

Figure 1: CT scan of the chest, abdomen and pelvis without contrast demonstrates evidence of prior ascending thoracic aortic repair and central displacement of atherosclerotic calcifications within the distal abdominal aorta.

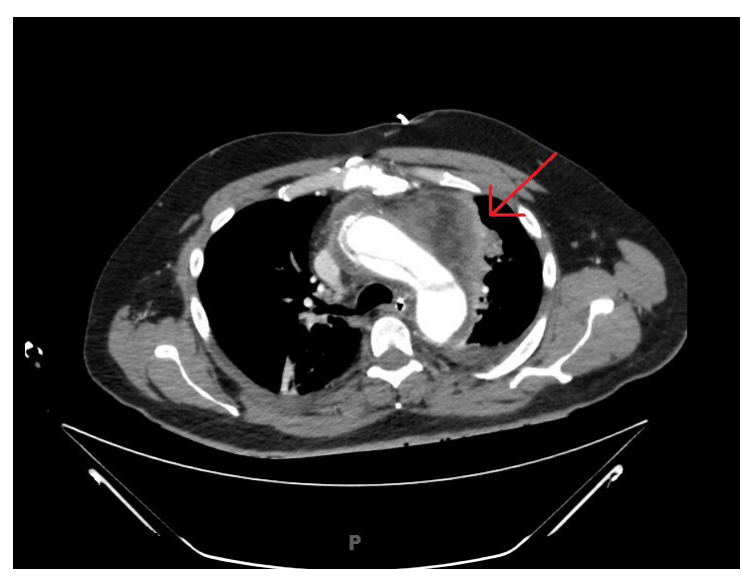

Figure 2: CT angiography of the chest shows fluid surrounding the ascending thoracic aorta and anterior to the trachea. Evidence of (prior) thoracoabdominal aortic dissection is visualized.

\section{Discussion}

Aortic abscess is a complication of IE which requires surgical intervention [4]. $22 \%$ of patients with aortic abscess have involvement of the aortic root, which is called aortic root abscess (ARA) or peri-annular abscess [4]. ARA can present in a multitude of ways, ranging from IE not improving with appropriate antibiotics to arrhythmia to syncope, rarely, ischemia due to coronary artery compression [5-7]. Similar to IE, not all patients with ARA will show specific signs and symptoms, often presenting with fever or malaise, so it is crucial to obtain a TEE even without obvious signs of ARA [8]. For instance, in a case reported by Landa et al. [5] a patient presented with right lower quadrant abdominal pain and general malaise. Initially, she was diagnosed and treated only for cholecystitis, but later during their hospital stay developed Wenckebach and a new diastolic murmur. They were subsequently diagnosed with ARA via TEE and had an aortic valve replacement along with laparoscopic cholecystectomy. It has been suggested that all patients with aortic valve endocarditis who do not improve within 72 hours on appropriate antibiotics should be evaluated for ARA specifically [7]. ARA can also manifest as a new onsetarrhythmia, tending to present with heart block-first, second, and third degree-so in patients with new-onset heart block with no other known explanation, ARA should be considered. Repeat physical exams during hospitalization to re-evaluate for signs of IE, as well as ARA, are also important [5].

IE, even without other complications, is a serious disease with inpatient mortality, estimated to be $20 \%$ [2]. The high mortality of IE is mostly attributed to perivalvular abscess formation and development of CHF. Additionally, IE of the aortic valve has higher mortality and is more associated with both higher incidences of abscess formation and CHF [1]. The importance of surgical intervention cannot be underestimated, with surgery decreasing 6-month mortality of complicated IE from an estimated 33\% to $16 \%$ [1]. Patients who develop ARA are often critically ill, many requiring urgent/emergent surgical intervention and often inotropic support or percutaneous cardiopulmonary support as well $[3,4]$. ARA has an even worse prognosis than prosthetic valve endocarditis (PVE), with intra-operative mortality of 19.7 as compared to 11.6 and 10year survival of $52 \%$ versus $62 \%$, respectively [4]. In a retrospective study of 108 patients with ARA by Siniawski et al. [9] the factors found to most closely indicate a poor prognosis were severe aortic root destruction and sepsis. Other factors found to be associated with higher morbidity were female gender, an early phase of the abscess, shock (defined as requiring pressor support), and poor LV 
function [9]. Factors identified by other studies as being associated with worse prognosis include the presence of a prosthetic valve, abscess size, and the presence of heart block [1,3,4]. Possible complications of ARA include heart block, valve destruction, fistula formation, aortic pseudoaneurysm formation, the involvement of the mitral valve, extrinsic compression of coronary arteries, PVE recurrence, para-prosthetic valve leaks, and valve degeneration [3-4]. In a retrospective study by Lee et al. [3] of the 49 patients with ARA, 5 developed a pseudoaneurysm and 4 developed fistulas. Fistula formation is one of the most feared complications, in part due to the practical considerations-ARAs with fistulas require extensive debridement, which can complicate the reconstruction process of the surgery later on [10].

Although it is agreed upon that surgery is the standard of care for patients with ARA, there has been some discussion about which approach has better outcomes. Most patients with ARA undergo an aortic valve replacement (AVR), although those who require more radical debridement, such as those with fistulas, undergo a total aortic root replacement (ARR). Some studies have indicated that ARR may have a better prognosis, although there is limited data. A retrospective analysis of 27 patients with ARA over 15 years indicated that there may be decreased complications associated with ARR [4]. 20 patients in this study received AVR while 7 received ARR [4]. The hospital mortality rate was higher for patients who received AVR versus ARR, at $25 \%$ and $14.3 \%$ respectively, but these results were not statistically significant, possibly related to the small sample size [4]. Another study, a meta-analysis comparing AVR versus ARR, found no difference in 30-day mortality of the 2 modalities [11]. However, ARR did have statistically significantly lower rates of re-operation within 1 year [11]. Choosing which modality is best for each individual patient is a highly complex and personalized decision, but it remains vital that these patients are recognized swiftly and referred for surgery. Patients can have a host of different presentations and it is important for the clinician to be aware of the multitude of ways in which patients present.

\section{Conclusion}

Like IE, ARA can prove a challenge to diagnose, given that many patients present with vague symptoms. However, IE has high morbidity and mortality, especially when complicated by ARA. Early diagnosis is crucial given that these patients benefit from early surgical intervention. Just as it is important to diagnose IE, it is crucial for clinicians to be aware of ARA and to evaluate patients with IE for ARA as well to prevent complications and better patient outcomes.

\section{Author's Contribution}

Gabriella A: Case selection, planning and drafting the manuscript.

Marjan Alidoost: Contributed to discussion and edition of the manuscript.

John Mikhail: Contributed in preparation and finalized the manuscript.

Hossain MA: Contributed in manuscript revision and final approval.

\section{Acknowledgment}

We thank our patient who kindly gave his consent for this publication.

\section{References}

1. Habib G, Lancellotti P, Antunes MJ, Bongiorni MG, Casaltaet JP, et al. (2015) 2015 ESC guidelines for the management of infective endocarditis: The task force for the management of infective endocarditis of the European society of cardiology (ESC) endorsed by: European association for cardio-thoracic surgery (EACTS), the European association of nuclear medicine (EANM). OUP Academic, Oxford University Press, UK.

2. Anna G, Jainandunsing JS, Van Assen S, Van Geel PP, Sinha B, et al. (2018) A standardized approach to treat complex aortic valve endocarditis: a case series. Journal of Cardiothoracic Surgery 13(1).

3. Lee, Sak, Chang BC, Park HK (2014) Surgical experience with infective endocarditis and aortic root abscess. Yonsei Med J 55(5): 1253-1259.

4. Kirali K, Sarikaya S, Ozen Y, Sacli H, Basaran E, et al. (2016) Surgery for aortic root abscess: A 15-Year experience. Tex Heart Inst J 43(1): 20-28.

5. Landa AB, Viswanath O, D Mello J (2018) Aortic root abscess and the lost art of the physical exam. Saudi J Anaesth 12(4): 654-656.

6. Misuraca L, DeCaro F, DeCarlo M, Barzaghi C, Scioti G, et al. (2012) Left main compression by an aortic root abscess. J Cardiovasc Med 13(3): 207-208.

7. Brecker SJD, Pepper JR, Eykyn SJ (1999) Aortic root abscess. Heart 82(3): 260-262.

8. Bindroo S, Akhter S, Thakur K, Geller C (2018) Austrian triad complicated by septic arthritis and aortic root abscess. Cureus 10(7): e3018.

9. Siniawski H, Grauhan O, Hofmann M, Pasic M, Weng Y, et al. (2005) Aortic root abscess and secondary infective mitral valve disease: Results of surgical endocarditis treatment. Eur J Cardiothorac Surg 27(3): 434-440.

10. Bozbuga N, Erentug V, Erdogan HB, Kirali K, Ardal H, et al. (2004) Surgical treatment of aortic abscess and fistula. Tex Heart Inst J 31(4): 382-386.

11. Chen GJ, Lo WC, Tseng HW, Pan SC, Chen YS, et al. (2017) Outcome of surgical intervention for aortic root abscess: A meta-analysis. Eur J Cardiothorac Surg 53(4): 807-814. 